\title{
A Game-Theoretic Analysis of Games with a Purpose
}

\section{Citation}

Jain, Shaili, and David C. Parkes. 2008. A game-theoretic analysis of games with a purpose. In Internet and Network Economics, ed. C. H Papadimitriou, S. Zhang, 342-350. Berlin: Springer. Previously published in Lecture Notes in Computer Science 5385: 342-350.

\section{Published Version}

doi:10.1007/978-3-540-92185-1_40

\section{Permanent link}

http://nrs.harvard.edu/urn-3:HUL.InstRepos:4000812

\section{Terms of Use}

This article was downloaded from Harvard University's DASH repository, and is made available under the terms and conditions applicable to Other Posted Material, as set forth at http:// nrs.harvard.edu/urn-3:HUL.InstRepos:dash.current.terms-of-use\#LAA

\section{Share Your Story}

The Harvard community has made this article openly available.

Please share how this access benefits you. Submit a story.

Accessibility 


\title{
A Game-Theoretic Analysis of Games with a Purpose
}

\author{
Shaili Jain David C. Parkes \\ School of Engineering and Applied Science \\ Harvard University \\ \{shailij, parkes\}@eecs.harvard.edu
}

\begin{abstract}
We present a simple game-theoretic model for the ESP game, an interactive game devised to label images on the web, and characterize the equilibrium behavior of the model. We show that a simple change in the incentive structure can lead to different equilibrium structure and suggest the possibility of formal incentive design in achieving desirable system-wide outcomes, complementing existing considerations of robustness against cheating and human factors.
\end{abstract}

\section{Introduction}

Showcased by the early success of "Games with a Purpose" [3], human computation considers the possibility that networks of people can be leveraged to solve large-scale problems that are hard for computers. Work by von Ahn and others has shown the tremendous power that networks of humans possess to solve problems while playing computer games $[4,7,5,6]$. The ESP game is an example of such human computation; it is an interactive system that allows users to be paired to play games that label images on the web [4]. Users play the ESP game because it is an enjoyable game to play, with the added side-effect that they are doing useful work in the process. Subsequent work to the ESP game has included Peekaboom [7], Phetch [5], and Verbosity [6]. Hsu and colleagues [2, 1] developed a simple game called PhotoSlap for determining content of images and provided a game-theoretic analysis for PhotoSlap.

While there has been incredible progress in the area of human computation, there is still much more potential. For "Games with a Purpose", it seems especially appropriate to use game theory to better understand how to design incentives in order to achieve system-wide goals. For example, it appears anecdotally that during play of the ESP game that people coordinate on easy words and that the game is less effective in labeling less obvious, harder words. Through this line of work, we ultimately aim to show that proper incentive design along with appropriate system design is an important paradigm for human computation and peer production problems.

This paper aims to study behavior in the ESP Game through a gametheoretic light. We propose a simple model of the game and consider two different models of payoffs, namely match-early preferences (MEP) and rare-words-first 
preferences. Match-early preferences model the setting in which players wish to complete as many rounds as possible and receive the same score irrespective of the words on which they match. The match-early preferences model is meant to reflect the current method of assigning scores to outcomes in the ESP game. Here we show that low effort is a Bayesian-Nash equilibrium for all distributions on word frequencies, with players focusing attention on high-frequency words. Rare-words-first preferences model the setting in which players wish to match on infrequent words before frequent words, we suppose because of appropriately designed incentives, and the speed with which a match is achieved is only a secondary consideration. We show that under this preference model, there is a significant difference in the equilibrium structure.

We briefly describe elements of the ESP game before introducing the model in the next section. In the ESP game, players are randomly paired with another player in the system for a set of 15 images. Players try to label as many images of the 15 as they can in the allotted 2.5 minutes. Players receive a fixed number of points after agreeing on a common word. In the set of 15 images, players get bonus points after agreeing on five images, ten images, and fifteen images in the same set. The only words that are used from the input streams are the first agreed word. An interesting feature of the ESP game, not modeled here, is the use of Taboo words [4]. Taboo words are words that are displayed next to the image that players cannot enter for the corresponding image. Taboo words are words that have been entered sufficiently many times in previous plays of the image and encourage players to enter different words so that the set of labels for an image can be extended. ${ }^{1}$ Modeling the effect of the Taboo Words is an important direction for future work.

\section{An ESP Model with Match-Early Preferences}

We model the ESP game as a two-player, two-stage game of imperfect information. We focus on modeling one of the 15 rounds, and thus the game associated with a specific image. We model the ESP game with each player sampling words from a universe of possible words associated with the picture, to which we associate a frequency ordering. Players can vary the effort level that relates to how likely they are to sample frequent words as opposed to infrequent words. Then players decide which order to play their sampled words in the game. In the model of match-early preferences, we instead capture the strategic behavior of having 15 rounds under a time constraint by providing a preference for matching in an earlier location than a later location.

Let $d>0$ denote the dictionary size, representing the number of words that each player will think of for the image at hand. We model a universe of words $U=\left\{w_{1}, w_{2}, \ldots, w_{n}\right\}$, that represents all possible words to describe the image and the knowledge that the game designer is trying to learn. Each word has an

\footnotetext{
${ }^{1}$ In addition, von Ahn and Dabbish came up with a number of methods to circumvent cheating, where possible methods of cheating include players trying to be paired with themselves and global strategies such as entering "a" for every image [4].
} 


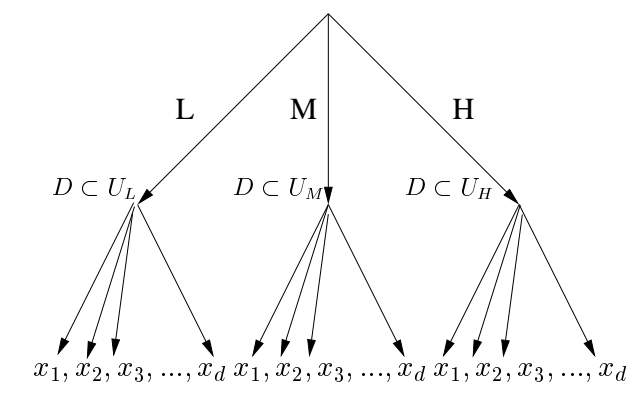

Fig. 1. The game tree above represents the decision space of one player

associated frequency, where $f_{i}$ denotes the frequency of word $w_{i}$. We assume that a player can rank the words sampled by frequency. The frequencies satisfy the property that $\sum_{i=1}^{n} f_{i}=1$. We assume that the words in the universe are ordered according to decreasing frequency, that is $f_{1} \geq f_{2} \geq \ldots \geq f_{n}$. The frequency of word $i$ can be considered the frequency that the word would be mentioned if a population of humans were each asked to state $d$ words related to the image. We assume that $1<d<|U|$.

Though this game has no communication between players and thus is properly analyzed as a normal-form game, it is useful to talk about a first stage (choosing an effort level) and a second stage (choosing a permutation on the dictionary). In the first stage of the game, players privately choose an effort level: $E=\{L, M, H\}$ for low, medium or high. The choice of effort level determines the set of words in the universe from which a player samples her dictionary. If a player chooses $L$ in the first stage of the game, the dictionary is sampled from the top $n_{L}>0$ words (without replacement). We say that a player that chooses effort level $L$ has universe $U_{L}$, where $U_{L}$ is exactly the set of the highest $n_{L}$ frequency words in $U$. If a player chooses effort $M$ in the first stage of the game, the dictionary is sampled from the top $n_{M}>n_{L}>0$ words. If a player chooses effort $H$, the dictionary is sampled from the top $n_{H}=n$ words (i.e., the entire universe). That is, word $i$ in $U$ is chosen with probability $f_{i, H}=f_{i}$.

Given word $x \in U, f_{e}(x)$ represents the frequency of word $x$ given the player has chosen effort level $e$. In Figure 1, this sample is modeled as a move by nature and can be considered to be the point at which a player learns her "type", namely her dictionary of words. Figure 1 represents the choices of a single player in the game, though both players are symmetric. Note that $n_{L}, n_{M}, n_{H}$, and $d$ are parameters of the model and there is no cost associated with each of the first level actions. We establish that low effort is an equilibrium under match-early preferences even without introducing a cost, which would increase with effort and presumably increase the benefits of low effort. We leave introducing cost into this model for future work.

In the second stage, once each player privately learns her dictionary based on the effort level chosen, players choose a permutation on the words. This models 
the decision about the order in which a player should enter words. This order on a player's dictionary defines the second-stage action of each player and determines the outcome of the game. The outcome is defined by the first word that is in the ordered-list of both players and the location (where the location is defined to be the maximum value of the two positions where the word occurs in each ordered-list). In what follows, we refer to $D_{1}$ as the dictionary for player 1 and $D_{2}$ as the dictionary for player 2 . The second stage strategy $s_{1} \in S_{1}$ for player 1 defines a specific order $s_{1}\left(D_{1}\right)$ on the words in $D_{1}$, for every possible dictionary. Likewise, player 2 has a second-stage strategy $s_{2} \in S_{2}$ that defines an order $s_{2}\left(D_{2}\right)$ for every dictionary. We restrict our attention to strategies that involve playing all words in the dictionary since any strategy that does not involve playing all words is weakly dominated by one that involves playing all words. We also restrict our attention to consistent strategies, strategies for a player that do not change the relative ordering of elements depending on the player's realized dictionary. In other words, a consistent strategy involves specifying a total ordering of elements on $U$ and applying that total ordering to the realized dictionary.

A complete strategy for the ESP game is an ordered pair $\sigma_{i}=\left(e_{i}, s_{i}\right) \in$ $E \times S_{i}=\Sigma_{i}$. This defines the play in both stages for all possible dictionaries. We focus here on pure strategies.

Definition 1. Suppose player 1 outputs a list of words $x_{1}, x_{2}, \ldots, x_{d}$ and player 2 outputs a list of words $y_{1}, y_{2}, \ldots, y_{d}$. If there exists $1 \leq i, j \leq d$ such that $x_{i}=y_{j}$, there was a match and this match occurred in location $\max (i, j)$. It is possible for two sequences to have more than one match, so we concern ourselves with the first match, that is the pair $i, j$ that minimizes $\max (i, j)$ such that $x_{i}=y_{j}$.

An outcome is an ordered pair $o=(w, l) \in(U \cup \phi) \times(\{1, \ldots, d\} \cup \phi)$ where $(\phi, \phi)$ indicates there was no match and the $(w, l)$ pair otherwise indicates that the first match on word $w \in U$ in location $l \in \mathcal{L}$ where $\mathcal{L}=\{1,2, \ldots, d\} \cup \phi$. Let $\mathcal{O}$ denote the set of possible outcomes. Since $s_{1}\left(D_{1}\right)$ and $s_{2}\left(D_{2}\right)$ specify orderings on given dictionaries, they induce an outcome: the location of the first match.

Let outcome function $g\left(s_{1}\left(D_{1}\right), s_{2}\left(D_{2}\right)\right) \in \mathcal{O}$ denote this outcome. The location (if any) of the first match is denoted by $g_{l}\left(s_{1}\left(D_{1}\right), s_{2}\left(D_{2}\right)\right) \in \mathcal{L}$.

Each player $i$ has valuation $v_{i}(o)$ on outcome $o$, which induces a (weak) total preference ordering on outcomes. For match-early preferences, we require $\left(w_{1}, l_{1}\right) \equiv\left(w_{2}, l_{1}\right) \equiv \ldots \equiv\left(w_{n}, l_{1}\right) \succ\left(w_{1}, l_{2}\right) \equiv\left(w_{2}, l_{2}\right) \equiv \ldots \equiv\left(w_{n}, l_{2}\right) \succ \ldots \succ$ $\left(w_{1}, l_{d}\right) \equiv\left(w_{2}, l_{d}\right) \equiv \ldots \equiv\left(w_{n}, l_{d}\right) \succ(\phi, \phi)$ for all players.

Let $\operatorname{Pr}\left(D_{i} \mid e_{i}\right)$ denote the probability of dictionary $D_{i}$ given effort level $e_{i}$. Often times we write this as $\operatorname{Pr}\left(D_{i}\right)$ and leave the effort level implicit.

Definition 2. The probability of first match in $l_{i}$ given $s_{1}\left(D_{1}\right), s_{2}$, and distribution $\operatorname{Pr}\left(D_{2}\right)$, is $p\left(l_{i}, s_{1}\left(D_{1}\right), s_{2}\right)=\sum_{D_{2}} \operatorname{Pr}\left(D_{2}\right) I\left(g_{l}\left(s_{1}\left(D_{1}\right), s_{2}\left(D_{2}\right)\right)=l_{i}\right)$. Similarly, the probability of first match in $l_{i}$ on $w_{j}$ is $p\left(w_{j}, l_{i}, s_{1}\left(D_{1}\right), s_{2}\right)=$ $\sum_{D_{2}} \operatorname{Pr}\left(D_{2}\right) I\left(g\left(s_{1}\left(D_{1}\right), s_{2}\left(D_{2}\right)\right)=\left(w_{j}, l_{i}\right)\right)$. Often times we will abbreviate $p\left(l_{i}, s_{1}\left(D_{1}\right), s_{2}\right)$ as $p\left(l_{i}\right)$ and $p\left(w_{j}, l_{i}, s_{1}\left(D_{1}\right), s_{2}\right)$ as $p\left(w_{j}, l_{i}\right)$. 
Let $u_{i}\left(s_{i}\left(D_{i}\right), s_{2-i}\left(D_{2-i}\right)\right)=v_{i}\left(g\left(s_{1}\left(D_{1}\right), s_{2}\left(D_{2}\right)\right)\right)$ be the utility of player $i$ given $D_{1}, D_{2}$. Let $u_{i}\left(s_{i}\left(D_{i}\right), s_{2-i}\right)=\sum_{D_{2-i}} \operatorname{Pr}\left(D_{2-i}\right) u_{i}\left(s_{i}\left(D_{i}\right), s_{2-i}\left(D_{2-i}\right)\right)$ be the expected (interim) utility of player $i$ given $D_{i}$ with respect to the distribution on all possible dictionaries of the other player, as induced by her effort level. Let $u_{i}\left(\sigma_{i}, \sigma_{2-i}\right)=\sum_{D_{1}} \sum_{D_{2}} \operatorname{Pr}\left(D_{1} \mid e_{1}\right) \operatorname{Pr}\left(D_{2} \mid e_{2}\right) u_{i}\left(s_{i}\left(D_{i}\right), s_{2-i}\left(D_{2-i}\right)\right)$ be the expected (ex ante) utility of player $i$ before dictionaries are sampled, given complete strategies $\sigma=\left(\sigma_{1}, \sigma_{2}\right)$.

We analyze the second stage of the game before analyzing the complete game. For this, consider the game induced by fixing top level effort levels $\left(e_{1}, e_{2}\right)$ for the two players (the second stage game conditioned on effort $e_{1}$ and $e_{2}$ ). In the second stage, each player knows her own dictionary but not the dictionary of the other player. We can now define two useful equilibrium concepts:

Definition 3. Strategy profile $s^{*}=\left(s_{1}^{*}, s_{2}^{*}\right)$ is an ex post Nash equilibrium of the second stage of the ESP game conditioned on effort levels $e_{1}$ and $e_{2}$, if for every $D_{1}$ and every $D_{2}$, we have:

$$
u_{i}\left(s_{i}^{*}\left(D_{i}\right), s_{2-i}^{*}\left(D_{2-i}\right)\right) \geq u_{i}\left(s_{i}^{\prime}\left(D_{i}\right), s_{2-i}^{*}\left(D_{2-i}\right)\right), \quad \forall s_{i}^{\prime} \neq s_{i}^{*}, \quad \forall i \in\{1,2\}
$$

Definition 4. Strategy profile $s^{*}=\left(s_{1}^{*}, s_{2}^{*}\right)$ is a strict Bayesian-Nash equilibrium of the second-stage of the ESP game conditioned on effort levels $e_{1}$ and $e_{2}$ if for both players $i \in\{1,2\}$, for every $D_{i}$,

$$
u_{i}\left(s_{i}^{*}\left(D_{i}\right), s_{2-i}^{*}\right)>u_{i}\left(s_{i}^{\prime}\left(D_{i}\right), s_{2-i}^{*}\right),
$$

where the probability adopted in interim utility $u_{i}$ for the distribution on the dictionary of player $2-i$ is induced by the effort of that player in the first stage.

Definition 5. Strategy profile $\sigma^{*}=\left(\sigma_{1}^{*}, \sigma_{2}^{*}\right) \in \Sigma_{1} \times \Sigma_{2}$ is a strict Bayesian-Nash equilibrium of the ESP game if for players $i \in\{1,2\}, u_{i}\left(\sigma_{i}^{*}, \sigma_{2-i}^{*}\right)>u_{i}\left(\sigma_{i}^{\prime}, \sigma_{2-i}^{*}\right)$.

Since the effort level chosen by each player is not visible to the other player, there is no need for a subgame perfect refinement.

\section{Effort Level of Players under Match-Early Preferences}

In this section, we analyze the equilibrium behavior under match-early preferences. We show that playing decreasing frequency in conjunction with low effort is a Bayesian-Nash equilibrium for the ESP game. First we see that playing playing words in order of decreasing frequency is not an ex-post Nash equilibrium for the second stage of the game.

Lemma 1. Suppose that players are playing the same effort level and there are three words in the universe, $w_{1}, w_{2}$, and $w_{3}$ with associated probabilities $f_{1, e}$, $f_{2, e}, f_{3, e}$, and with $f_{1, e}>f_{2, e}>f_{3, e}$. The second stage strategy profile $s=$ $\left(s_{1}, s_{2}\right)$, where $s_{1}$ and $s_{2}$ are the strategies of playing words in order of decreasing frequency, is not an ex-post Nash equilibrium. 
Since playing words in order of decreasing frequency is not an ex-post Nash equilibrium, we focus instead on Bayesian-Nash equilibrium. The following definition of stochastic dominance will enable equilibrium analysis for any valuation function that satisfies MEP. In Lemmas 2 and 3, we show that this notion of stochastic dominance is both sufficient and necessary for utility maximization.

Definition 6. Fixing effort levels $e_{1}$ and $e_{2}$ and fixing opponent's secondstage strategy $s_{2}$, we say second-stage strategy $s_{1}$ with match vector $\left(p\left(l_{1}, s_{1}\left(D_{1}\right), s_{2}\right), p\left(l_{2}, s_{1}\left(D_{1}\right), s_{2}\right), \ldots, p\left(l_{d}, s_{1}\left(D_{1}\right), s_{2}\right)\right)$ stochastically dominates second-stage strategy $s_{1}^{\prime}$ with match vector $\left(p\left(l_{1}, s_{1}^{\prime}\left(D_{1}\right), s_{2}\right), p\left(l_{2}, s_{1}^{\prime}\left(D_{1}\right), s_{2}\right)\right.$, $\left.\ldots, p\left(l_{d}, s_{1}^{\prime}\left(D_{1}\right), s_{2}\right)\right)$ with respect to dictionary $D_{1}$ if for every $1 \leq k \leq d$, $\sum_{a=1}^{k} p\left(l_{a}, s_{1}\left(D_{1}\right), s_{2}\right) \geq \sum_{a=1}^{k} p\left(l_{a}, s_{1}^{\prime}\left(D_{1}\right), s_{2}\right)$. We say that the stochastic dominance property is strict if there exists a $k$ such that $1 \leq k \leq d$ and $\sum_{a=1}^{k} p\left(l_{a}, s_{1}\left(D_{1}\right), s_{2}\right)>\sum_{a=1}^{k} p\left(l_{a}, s_{1}^{\prime}\left(D_{1}\right), s_{2}\right)$.

Lemma 2. If strategy $s_{1}$ stochastically dominates strategy $s_{1}^{\prime}$ with respect to dictionary $D_{1}$, for fixed opponent strategy $s_{2}$, then $u_{1}\left(s_{1}\left(D_{1}\right), s_{2}\right) \geq u_{1}\left(s_{1}^{\prime}\left(D_{1}\right), s_{2}\right)$, for all valuations consistent with match-early preferences.

Lemma 3. If $u_{1}\left(s_{1}\left(D_{1}\right), s_{2}\right) \geq u_{1}\left(s_{1}^{\prime}\left(D_{1}\right), s_{2}\right)$ for all valuations that are consistent with match-early preferences, then strategy $s_{1}$ must stochastically dominate strategy $s_{1}^{\prime}$ with respect to $D_{1}$, for fixed opponent strategy $s_{2}$.

Lemmas 2 and 3 can be extended to show strict stochastic dominance implies strictly greater utility and vice versa, for all valuations consistent with MEP.

Lemma 4. Given player 2 plays her words in order of decreasing frequency, the probability of first match in location $l_{1}$ is strictly maximized when player 1 plays her most frequent word first, for all dictionaries $D_{1}$ and effort levels $e_{2}$.

Lemma 5. For $1 \leq k<d$, given player 1 played her $k$ highest frequency words first and player 2 plays her words in order of decreasing frequency, the probability of first match in locations $l_{1}, \ldots, l_{k}$ is strictly maximized when player 1 plays her $k+1^{\text {st }}$ highest frequency word next, for all dictionaries $D_{1}$ and effort levels $e_{2}$.

Lemmas 2, 4, and 5 establish that playing decreasing frequency is a strict best response to an opponent who plays decreasing frequency.

Theorem 1. The strategy profile consisting of players playing words in order of decreasing frequency (denoted $(\downarrow, \downarrow)$ ) is a strict Bayesian-Nash equilibrium of the second stage of the ESP game, conditioned on any choice of effort levels $e_{1}$ and $e_{2}$, for any distribution over $U$ and any valuation function that satisfies match-early preferences.

To show that playing $L$ at the top-level along with playing decreasing frequency is a Bayesian-Nash equilibrium, we use the following definition of stochastic dominance for the top level of the game which fixes the equilibrium strategy for the bottom-level. The definition uses the following notation for a $k$-truncation of dictionary $D: D(k)$ is the set of $k$ highest frequency words in $D$. 
Definition 7. Fixing player 2's complete strategy $\left(e_{2}, s_{2}\right)$, a complete strategy $\left(e_{1}, s_{1}\right)$ stochastically dominates complete strategy $\left(e_{1}^{\prime}, s_{1}\right)$ for player 1 if:

$$
\begin{aligned}
& \sum_{D_{1, e_{1}}} \operatorname{Pr}\left(D_{1, e_{1}} \mid e_{1}\right) \sum_{D_{2, e_{2}}} \operatorname{Pr}\left(D_{2, e_{2}} \mid e_{2}\right) \cdot I\left(g_{l}\left(s_{1}\left(D_{1, e_{1}}(k)\right), s_{2}\left(D_{2, e_{2}}(k)\right)\right)=l_{1}, \ldots, l_{k}\right) \geq \\
& \sum_{D_{1, e_{1}^{\prime}}} \operatorname{Pr}\left(D_{1, e_{1}^{\prime}} \mid e_{1}^{\prime}\right) \sum_{D_{2, e_{2}}} \operatorname{Pr}\left(D_{2, e_{2}} \mid e_{2}\right) \cdot I\left(g_{l}\left(s_{1}\left(D_{1, e_{1}^{\prime}}(k)\right), s_{2}\left(D_{2, e_{2}}(k)\right)\right)=l_{1}, \ldots, l_{k}\right) \forall k
\end{aligned}
$$

where $g_{l}\left(s_{1}\left(D_{1, e_{1}}(k)\right), s_{2}\left(D_{2, e_{2}}(k)\right)\right)$ gives the outcome when second-stage strategies $s_{1}$ and $s_{2}$ act on $D_{1, e_{1}}(k)$ and $D_{2, e_{2}}(k)$ and $I(\cdot)$ is the indicator function. The dominance is strict if there exists a $k$ such that the above inequality is strict.

Since Theorem 1 establishes that $(\downarrow, \downarrow)$ is a strict Bayesian-Nash equilibrium of the second stage, for all effort levels, we set $\left(s_{1}, s_{2}\right)=(\downarrow, \downarrow)$ and we know that $I\left(g_{l}\left(s_{1}\left(D_{1, e_{1}}(k)\right), s_{2}\left(D_{2, e_{2}}(k)\right)\right)=l_{1}, \ldots, l_{k}\right)=I\left(D_{1, e_{1}}(k) \cap D_{2, e_{2}}(k) \neq \emptyset\right)$. Similar to Lemmas 2 and 3, we can show that stochastic dominance in Definition 7 is sufficient and necessary for utility maximization.

In order to establish stochastic dominance, we construct a randomized mapping for each dictionary that can be sampled when playing $M$ to a number of dictionaries that can be sampled when playing $L$. Each dictionary in $\mathcal{D}_{M}$ is mapped to a dictionary in $\mathcal{D}_{L}$ that is at least as likely to match against the opponent's dictionary, averaged over the distribution of all possible dictionaries for the opponent. This is shown in Lemma 6 . In order to complete the proof, it is necessary to show that under the randomized mapping, no element in $\mathcal{D}_{L}$ is mapped to with greater probability under the randomized mapping than under the original distribution over $\mathcal{D}_{L}$. This fact is shown in Lemma 7 .

The randomized mapping $h$ can be described as follows: Consider a dictionary $D \in \mathcal{D}_{M}, D=A \cup B$, where $A$ is the set of "low words" and $B$ is the set of "medium words" (in other words, $A=D \cap U_{L}$ and $B=D \cap\left(U_{M}-U_{L}\right)$ ). Under our randomized mapping, $D$ is mapped to all dictionaries in $D_{L} \in \mathcal{D}_{L}$ such that $A \subset D_{L}$. In other words, $D$ is mapped to dictionary $D_{L} \in \mathcal{D}_{L}$ with non-zero probability if and only if $A \subset D_{L}$. If $A \subset D_{L}$, then $D$ is mapped to $D_{L}$ with the same probability that you could would get $D_{L}$ if you continued to sample individual words from $U_{M}$ (without replacement) until you got $d$ "low words". Note that if $D$ contains only medium words, $D$ is mapped to all dictionaries in $\mathcal{D}_{L}$ with non-zero probability. Likewise, if $D$ contains only low words, $D$ is mapped to only one dictionary in $\mathcal{D}_{L}$.

Lemma 6. For any $D_{1, M}$, where $D_{1, M}$ is a dictionary sampled with respect to the $M$ effort level, for any $h$ that satisfies the property that $D_{1, M}$ is mapped to a dictionary in $\mathcal{D}_{L}$ that contains the set $D_{1, M} \cap U_{L}$, any effort level of player 2 and when players play decreasing frequency in the second stage, we have that:

$$
\begin{aligned}
& \sum_{D_{2}} \operatorname{Pr}\left(D_{2}\right) \cdot I\left(h\left(D_{1, M}\right)(k) \cap D_{2}(k) \neq \emptyset\right) \geq \\
& \sum_{D_{2}} \operatorname{Pr}\left(D_{2}\right) \cdot I\left(D_{1, M}(k) \cap D_{2}(k) \neq \emptyset\right) \forall k \text { and } D_{1, M}
\end{aligned}
$$


In addition, the inequality is strict for all $k>k^{\prime}$ when $h\left(D_{1, M}\right) \neq D_{1, M}$ and $k^{\prime}$ is the first coordinate where $h\left(D_{1, M}\right)$ and $D_{1, M}$ differ.

Lemma 7 states the distribution obtained from sampling $U_{L}$ directly is the same as the distribution obtained from sampling a medium dictionary, followed by the randomized mapping (sampling $U_{M}$ until you get $d$ low words).

Lemma 7. $\operatorname{Pr}\left(D_{1, L} \mid L\right)=\sum_{D_{1, M}} \operatorname{Pr}\left(D_{1, M} \mid M\right) \cdot \operatorname{Pr}\left(h\left(D_{1, M}\right)=D_{1, L}\right)$

Lemma 8 uses Lemmas 6 and 7 to show that playing $L$ stochastically dominates playing $M$, assuming players play decreasing frequency in the second stage. An identical argument can be used to show that playing $L$ stochastically dominates playing $H$, assuming players play decreasing frequency in the second stage.

Lemma 8. For any effort level $e_{2}$ and when players play decreasing frequency in the second stage:

$$
\begin{aligned}
& \sum_{D_{1, L}} \operatorname{Pr}\left(D_{1, L} \mid L\right) \sum_{D_{2}} \operatorname{Pr}\left(D_{2}\right) \cdot I\left(D_{1, L}(k) \cap D_{2}(k) \neq \emptyset\right)> \\
& \sum_{D_{1, M}} \operatorname{Pr}\left(D_{1, M} \mid M\right) \sum_{D_{2}} \operatorname{Pr}\left(D_{2}\right) \cdot I\left(D_{1, M}(k) \cap D_{2}(k) \neq \emptyset\right) \forall k
\end{aligned}
$$

Theorem 1 together with Lemma 8 give us the following result.

Theorem 2. $((L, \downarrow),(L, \downarrow))$ is a strict Bayesian-Nash equilibrium for the complete game. Additionally, $(L, \downarrow)$ is a strict best-response to both $(M, \downarrow)$ and $(H, \downarrow)$.

\section{The Effect of Rare-Words First Preferences}

In this section, we consider the effect of modified preferences. We introduce a new model called rare-words first preferences and show some initial results regarding how equilibrium behavior is different under this new model.

Definition 8. Under rare-words first preferences, players prefer to match on rare words, with location as a secondary consideration. Any valuation function $v(o)$ that satisfies rare-words first preferences satisfies the following total ordering on outcomes: $\left(w_{n}, l_{1}\right) \succ\left(w_{n}, l_{2}\right) \succ \ldots \succ\left(w_{1}, l_{d-1}\right) \succ\left(w_{1}, l_{d}\right) \succ(\phi, \phi)$.

This preference relation allows for a virtually identical definition of stochastic dominance as Definition 6 which in turn leads to results analogous to Lemmas 2 and 3, namely that stochastic dominance is both sufficient and necessary for utility maximization.

The following lemma is in stark contrast with the results in section 4, where we showed that $(\downarrow, \downarrow)$ is a strict Bayesian-Nash equilibrium in the second stage, for all distributions over $U$, all valuation functions that satisfy MEP, and any pair of effort levels. Lemma 9 shows that we cannot say $(\downarrow, \downarrow)$ is a BayesianNash equilibrium for the second stage of the game for any distribution, without making more assumptions on the valuation function. 
Lemma 9. Consider any distribution over $U=\left\{w_{1}, w_{2}, \ldots, w_{n}\right\}$ and suppose that player 2 is playing her words in order of decreasing frequency. For any dictionary of player 1, no consistent strategy of player 1 can stochastically dominate all other consistent strategies.

Similarly, Lemma 10 shows that when a player is playing increasing frequency, we need to make more assumptions on the valuation function to discern the bestresponse in the space of consistent strategies.

Lemma 10. Consider any distribution over $U=\left\{w_{1}, w_{2}, \ldots, w_{n}\right\}$ and suppose that player 2 is playing her words in order of increasing frequency. For any dictionary of player 1, no consistent strategy of player 1 can stochastically dominate all other consistent strategies.

We leave it as future work to characterize the set of sufficient and necessary conditions for which playing words in order of increasing frequency in conjunction with high effort for both players is a Bayesian-Nash equilibrium. Understanding the incentive structure that leads to high effort is important since it is one way the system designer can extend the set of labels for an image. In addition to this, future work should identify specific score functions that provide desirable equilibrium, and also move to analyze the larger context of a system of bilateral games with a view on understanding methods to induce large-scale desirable behavior, including such aspects as formalizing the role of Taboo Words and leveraging the entire sequence of words suggested by a player rather than just the particular match.

\section{References}

1. T.-H. Chang, C.-J. Ho, and J. Y. jen Hsu. The photoslap game: Play to annotate. In Proceedings of the Twenty-Second Conference on Artificial Intelligence (AAAI), pages 1966-1967, 2007.

2. C.-J. Ho, T.-H. Chang, and J. Y. jen Hsu. Photoslap: A multi-player online game for semantic annotation. In Proceedings of the Twenty-Second Conference on Artificial Intelligence (AAAI), pages 1359-1364, 2007.

3. L. von Ahn. Games with a purpose. IEEE Computer, 39(6):92-94, 2006.

4. L. von Ahn and L. Dabbish. Labeling images with a computer game. In Proceedings of the 2004 Conference on Human Factors in Computing Systems (CHI), pages 319-326, 2004.

5. L. von Ahn, S. Ginosar, M. Kedia, R. Liu, and M. Blum. Improving accessibility of the web with a computer game. In Proceedings of the 2006 Conference on Human Factors in Computing Systems (CHI), pages 79-82, 2006.

6. L. von Ahn, M. Kedia, and M. Blum. Verbosity: a game for collecting commonsense facts. In Proceedings of the 2006 Conference on Human Factors in Computing Systems (CHI), pages 75-78, 2006.

7. L. von Ahn, R. Liu, and M. Blum. Peekaboom: a game for locating objects in images. In Proceedings of the 2006 Conference on Human Factors in Computing Systems (CHI), pages 55-64, 2006. 